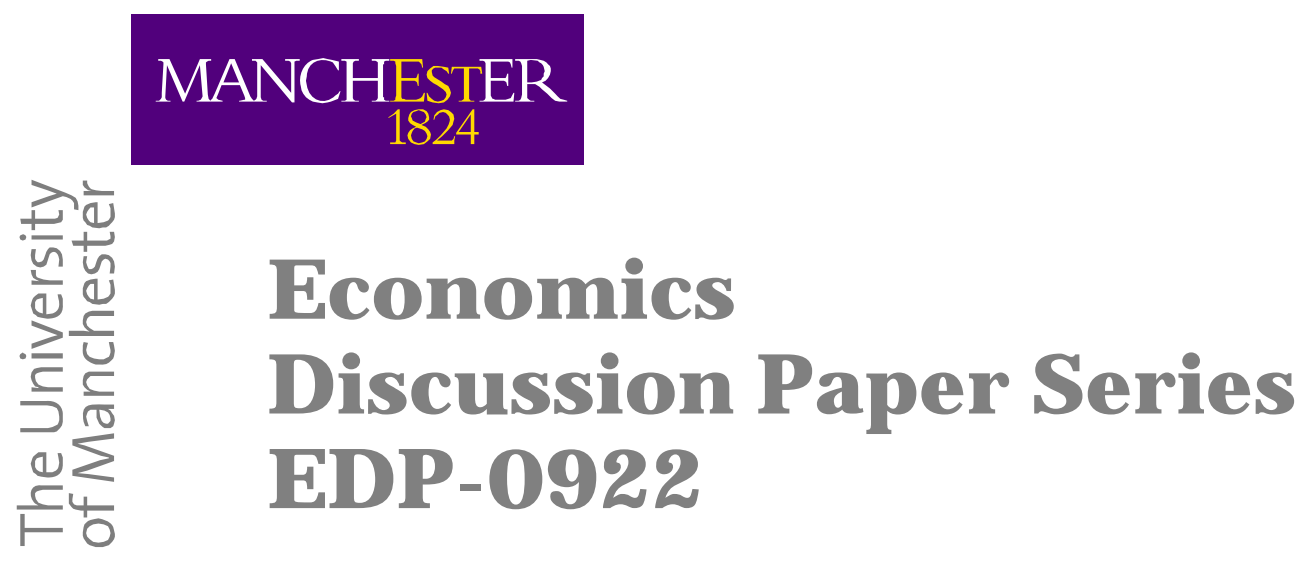

\title{
Existence of an interim and ex-ante minimax point for an asymmetric information game
}

\author{
Marialaura Pesce \\ Nicholas C. Yannelis
}

November 2009

Economics

School of Social Sciences

The University of Manchester

Manchester M13 9PL 


\title{
Existence of an interim and ex-ante minimax point for an asymmetric information game*
}

November 13, 2009

Marialaura Pesce ${ }^{1}$ and Nicholas C. Yannelis ${ }^{2}$

\begin{abstract}
We introduce the notions of ex-ante and interim minimax point for an asymmetric information game and prove the existence of such points. Our new results include as a special case the theorem in [2].
\end{abstract}

${ }^{*}$ This paper was conceived via discussions with Roko Aliprantis. We are grateful to him for introducing us to the existence of a minimax problem. We are also thankful to Iryna Topolyan for useful comments.

${ }^{1}$ Dipartimento di Matematica e Statistica, Universitá di Napoli Federico II, Napoli 81026, ITALY

e-mail: marialaura.pesce@unina.it

${ }^{2}$ Department of Economics

University of Illinois at Urbana-Champaign, Urbana, IL 61801, USA and

Economics - School of Social Sciences

The University of Manchester, Oxford Road, Manchester M13 9PL, UK

e-mail: nyanneli@illinois.edu, nicholasyannelis@manchester.ac.uk 


\section{Introduction}

In a recent paper Aliprantis et al. [2] provide an existence proof of a minimax point for a strategic (normal) form game. This theorem is useful as it has found applications in game theory (see for example Fudenberg-Maskin [5], Myerson [6], Thomas [7] among others).

Our objective is to extend the above result of Aliprantis et al. [2] to an asymmetric information game. To this end we introduce the notions of ex-ante and interim minimax point and prove the existence of such points.

Although the idea of the proof is the same with that in [2] the introduction of asymmetric information necessitates the use of some non trivial theorems. In particular, we make use of Diestel's theorem on weak compactness on the space of Bochner integrable functions and the Kuratowski measurable selection theorem.

As the deterministic result proved in [2] has found interesting applications in repeated games, we think that our new results will be of interest and applicable to a framework of repeated games with asymmetric information. Obviously, our new general setting includes as a special case the result in [2].

\section{Mathematical preliminaries}

\section{$2.1 \quad$ Notation}

$\mathbb{R}$ denotes the set of real numbers.

$\mathbb{R}_{+}=\{x \in \mathbb{R}: x \geq 0\}$.

$\mathbb{R}_{++}=\{x \in \mathbb{R}: x>0\}$.

$\overline{\mathbb{R}}=\mathbb{R} \cup\{-\infty,+\infty\}$.

$2^{A}$ denotes the set of all subsets of the set $A$.

\subsection{Definitions}

If $\mathrm{X}$ and $\mathrm{Y}$ are sets, the graph of the set-valued function (or correspondence), $\Phi: X \rightarrow 2^{Y}$ is denoted by $G_{\Phi}=\{(x, y) \in X \times Y: y \in \Phi(x)\}$. Let $(T, \mathcal{T}, \mu)$ be a complete, finite measure space, and $X$ be a separable Banach space. The set-valued function $\Phi: T \rightarrow 2^{X}$ is said to have a measurable graph if $G_{\Phi} \in \mathcal{T} \otimes \beta(X)$, where $\beta(X)$ denotes the Borel $\sigma$-algebra on $X$ and $\otimes$ denotes the product $\sigma$-algebra. A correspondence $\Phi: T \rightarrow 2^{X}$ from a measurable space $(T, \mathcal{T}, \mu)$ into a topological space $X$ is said to be lower measurable if $\{t \in T: \Phi(t) \cap V \neq \emptyset\} \in \mathcal{T}$ for every $V$ open in $X$.

If $X$ and $Y$ are topological spaces, $\Phi: X \rightarrow Y$ is said to be upper-semi-continuous if the set $\{x \in X: \Phi(x) \subset V\}$ is open in $X$ for every open subset $V$ of $Y$; $\Phi$ is said to be lower 
semi-continuous if the set $\{x \in X: \Phi(x) \cap V \neq \emptyset\}$ is open in $X$ for every open subset $V$ of $Y$.

Let $(T, \mathcal{T}, \mu)$ be a finite measure space and $X$ be a Banach space. Following Diestel-Uhl (1977) (see [4]) the function $f: T \rightarrow X$ is called simple if there exist $x_{1}, x_{2}, \ldots, x_{n}$ in $X$ and $\alpha_{1}, \alpha_{2}, \ldots, \alpha_{n}$ in $\mathcal{T}$ such that $f=\sum_{i=1}^{n} x_{i} \chi_{\alpha_{i}}$, where $\chi_{\alpha_{i}}(t)=1$ if $t \in \alpha_{i}$ and $\chi_{\alpha_{i}}(t)=0$ if $t \notin \alpha_{i}$. A function $f: T \rightarrow X$ is said to be $\mu$-measurable if there exists a sequence of simple functions $f_{n}, T \rightarrow X$ such that $\lim _{n \rightarrow \infty}\left\|f_{n}(t)-f(t)\right\|=0$ for almost all $t \in T$. A $\mu$-measurable function $f: T \rightarrow X$ is said to be Bochner integrable if there exists a sequence of simple functions $\left\{f_{n}: n=1,2, \ldots\right\}$ such that

$$
\lim _{n \rightarrow+\infty} \int_{T}\left\|f_{n}(t)-f(t)\right\| d \mu(t)=0 .
$$

In this case we define for each $E \in \mathcal{T}$ the integral to be $\int_{E} f(t) d \mu(t)=\lim _{n \rightarrow+\infty} \int_{E} f_{n}(t) d \mu(t)$. It can be shown [see Diestel-Uhl (1977), Theorem 2, p.45 [4]] that if $f: T \rightarrow X$ is a $\mu$ measurable function, then $f$ is Bochner integrable if and only if $\int_{T}\|f(t)\| d \mu(t)<\infty$. It is important to note that the Dominated Convergence Theorem holds for Bochner integrable functions. In particular, if $f_{n}: T \rightarrow X,(n=1,2, \ldots)$ is a sequence of Bochner integrable functions such that $\lim _{n \rightarrow+\infty} f_{n}(t)=f(t)$ for almost all $t \in T$, and $\left\|f_{n}(t)\right\| \leq g(t)$ for almost all $t \in T$ (where $g: T \rightarrow \mathbb{R}$ is an integrable function), then $f$ is Bochner integrable and $\lim _{n \rightarrow+\infty} \int_{T}\left\|f_{n}(t)-f(t)\right\| d \mu(t)=0$.

For $1 \leq p<\infty$, we denote by $L_{p}(\mu, X)$ the space of equivalence classes of $X$-valued Bochner integrable functions $x: T \rightarrow X$ normed by

$$
\|x\|_{p}=\left(\int_{T}\|x(t)\|^{p} d \mu\right)^{\frac{1}{p}} .
$$

It is a standard result that normed by the functional $\|\cdot\|$ above, $L_{p}(\mu, X)$ becomes a Banach space [see [4], p. 50]. Recall that a correspondence $\Phi: T \rightarrow 2^{X}$ is said to be integrably bounded if there exists a map $h \in L_{1}(\mu, \mathbb{R})$ such that $\sup \{\|x\|: x \in \Phi(t)\} \leq h(t)$ for almost all $t \in T$.

A Banach space $\mathrm{X}$ has the Radon-Nikodym Property with respect to the measure space $(T, \mathcal{T}, \mu)$ if for each $\mu$-continuous measure $G: \mathcal{T} \rightarrow X$ of bounded variation there exists $g \in L_{1}(\mu, X)$ such that $G(E)=\int_{E} g(t) d \mu(t)$ for all $E \in \mathcal{T}$.

$X^{*}$ stands for the topological dual space of $(X,\|\cdot\|)$. As usual $\|\cdot\|^{*}$ stands for the norm dual on $X^{*}$ (i.e., $\left\|x^{*}\right\|^{*}:=\sup \left\{<x, x^{*}>: x \in X,\|x\| \leq 1\right\}$, where $\left\langle x, x^{*}>:=\right.$ $\left.x^{*}(x)\right)$. By weak compactness and weak continuity we mean with respect to the weak topology $\sigma\left(L_{1}(\mu, X), L^{\infty}\left(\mu, X^{*}\right)\right)$, where $L^{\infty}\left(\mu, X^{*}\right)$ is the dual of $\left(L_{1}(\mu, X),\|\cdot\|_{1}\right)$. Recall 
that $\sigma\left(L_{1}(\mu, X), L^{\infty}\left(\mu, X^{*}\right)\right)$ is defined as the weakest topology on $L_{1}(\mu, X)$ for which all functionals

$$
x \in L_{1}(\mu, X) \rightarrow \int_{\Omega}<x(\omega), p(\omega)>d \mu, \quad \text { where } p \in L^{\infty}\left(\mu, X^{*}\right),
$$

are continuous.

\subsection{Theorems}

We now collect some interesting results, which will be of fundamental importance in this paper. We begin with a result on weak compactness known as Diestel's Theorem. This theorem is a consequence of the celebrated theorem by James on weakly compact sets.

Diestel's Theorem: Let $T$ be an arbitrary measure space, $X$ be a Banach space and $K: T \rightarrow 2^{X}$ be an integrably bounded, non-empty, weakly compact and convex valued correspondence. Then, the set

$$
\tilde{K}=\left\{f \in L_{1}(\mu, X): f(t) \in K(t) \text { for almost all } t \in T\right\}
$$

is weakly compact in $L_{1}(\mu, X)$.

Proof: see Yannelis (1991).

Kuratowski and Ryll-Nardzewski Measurable Selection Theorem Let $(T, \mathcal{T})$ be a measurable space, $X$ be a separable metric space and $\Phi: T \rightarrow 2^{X}$ be lower measurable, closed, non-empty valued correspondence. The, there exists a measurable function $f: T \rightarrow X$ such that $f(t) \in \Phi(t)$ for all $t \in T$.

Proof: See Castaing and Valadier [3].

Berge's Maximum Theorem Let $X$ be a topological space and $\Phi: T \rightarrow 2^{X}$ be a continuous correspondence with non-empty compact values, and suppose that $f: T \times X \rightarrow$ $\mathbb{R}$ is continuous. Define the "value function" $m: T \rightarrow \mathbb{R}$ by

$$
m(t)=\max _{x \in \Phi(t)} f(t, x),
$$

and the correspondence $\mu: T \rightarrow 2^{X}$ of maximizers by

$$
\mu(t)=\{x \in \Phi(t): f(t, x)=m(t)\} .
$$

Then, the value function $m$ is continuous, and the "argmax" correspondence $\mu$ is upper semi-continuous with compact values. 


\section{The strategic game with asymmetric information}

Let $(\Omega, \mathcal{F}, \mu)$ be a finite measure space denoting the states of nature of the world, let $I$ be the set of players, which may be any finite or infinite set, and $Y$ be a separable Banach space denoting the strategy sets. A strategic game with asymmetric information $G=\left\{\left(X_{i}, \mathcal{F}_{i}, u_{i}, q_{i}\right)_{i \in I}\right\}$ is a set of quadruples $\left(X_{i}, \mathcal{F}_{i}, u_{i}, q_{i}\right)$, where for each player $i$,

1. $X_{i}: \Omega \rightarrow 2^{Y}$ is the random strategy set,

2. $\mathcal{F}_{i}$ is a measurable partition ${ }^{3}$ of $(\Omega, \mathcal{F})$ denoting the private information of player $i$

3. $u_{i}: \Omega \times \prod_{i \in I} Y \rightarrow \mathbb{R}$ is the random payoff function,

4. $q_{i}: \Omega \rightarrow \mathbb{R}_{++}$is the prior of player $i$ (i.e., $q_{i}$ is a Radon-Nikodym derivative having the property that $\left.\int_{\omega \in \Omega} q_{i}(\omega) d \mu(\omega)=1\right)$.

As usual if $x \in \prod_{i \in I} Y$, then for each player $i$, we write $x=\left(x_{-i}, x_{i}\right)$, where $x_{-i}=$ $\left(x_{1}, \ldots, x_{i-1}, x_{i+1}, \ldots\right)$.

The $\sigma$-field of events discernable by every player is the "coarse" $\sigma$-field $\bigwedge_{i \in I} \mathcal{F}_{i}$, which is the largest $\sigma$-algebra contained in each $\mathcal{F}_{i}$. While, players by pooling their information, discern the events in the "fine" $\sigma$-field $\bigvee_{i \in I} \mathcal{F}_{i}$, which denotes the smallest $\sigma$-algebra containing all $\mathcal{F}_{i}$.

We denote by $L_{1}(\mu, Y)$ the space of equivalence classes of $Y$-valued Bochner integrable functions $x: \Omega \rightarrow Y$.

For each player $i \in I$ define the set of all Bochner integrable and $\mathcal{F}_{i}$ measurable selections from the strategy set of player $i$, i.e.,

$L_{X_{i}}=\left\{x_{i} \in L_{1}(\mu, Y): \quad x_{i}(\cdot)\right.$ is $\mathcal{F}_{i}$-measurable and $x_{i}(\omega) \in X_{i}(\omega)$ for almost all $\left.\omega \in \Omega\right\}$,

and let $L_{X}=\prod_{i \in I} L_{X_{i}}$. Similarly, for each player $i \in I$, we define $L_{X_{-i}}=\prod_{j \in I \backslash\{i\}} L_{X_{j}}$ and we notice that for each $i, L_{X}=L_{X_{-i}} \times L_{X_{i}}$.

For each player $i$, the ex-ante expected payoff $v_{i}: L_{X_{-i}} \times L_{X_{i}} \rightarrow \mathbb{R}$ is defined by

$$
v_{i}\left(x_{-i}, x_{i}\right)=\int_{\omega \in \Omega} u_{i}\left(\omega, x_{-i}(\omega), x_{i}(\omega)\right) q_{i}(\omega) d \mu(\omega) .
$$

\footnotetext{
${ }^{3}$ By an abuse of notation we will still denote by $\mathcal{F}_{i}$ the $\sigma$-algebra that the partition $\mathcal{F}_{i}$ generates.
} 
For each player $i$, denote by $E^{\mathcal{F}_{i}}(\omega)$ the event in $\mathcal{F}_{i}$ containing the realized state of nature $\omega$. Suppose that for all $\omega, \int_{\omega^{\prime} \in E^{\mathcal{F}_{i}(\omega)}} q_{i}\left(\omega^{\prime}\right) d \mu\left(\omega^{\prime}\right)>0$. For each player $i$, given $E^{\mathcal{F}_{i}}(\omega)$, the interim expected payoff $V_{i}: \Omega \times L_{X_{-i}} \times L_{X_{i}} \rightarrow \mathbb{R}$ is defined by

$$
V_{i}\left(\omega, x_{-i}, x_{i}\right)=\int_{\omega^{\prime} \in E^{\mathcal{F}_{i}}(\omega)} u_{i}\left(\omega^{\prime}, x_{-i}\left(\omega^{\prime}\right), x_{i}\left(\omega^{\prime}\right)\right) q_{i}\left(\omega^{\prime} \mid E^{\mathcal{F}_{i}}(\omega)\right) d \mu\left(\omega^{\prime}\right),
$$

where

$$
q_{i}\left(\omega^{\prime} \mid E^{\mathcal{F}_{i}}(\omega)\right)= \begin{cases}0 & \text { if } \omega^{\prime} \notin E^{\mathcal{F}_{i}}(\omega) \\ \frac{q_{i}\left(\omega^{\prime}\right)}{\int_{\omega^{\prime} \in E^{\mathcal{F}_{i}(\omega)}} q_{i}\left(\omega^{\prime}\right) d \mu\left(\omega^{\prime}\right)} & \text { if } \omega^{\prime} \in E^{\mathcal{F}_{i}}(\omega) .\end{cases}
$$

\section{Definitions of ex-ante and interim minimax point}

We now introduce the definitions of a minimax point in the context of an asymmetric information game, by considering the ex-ante as well as the interim case.

The minimax payoff of player $i$ gives the maximal punishment that all the other players can inflict on him. Using the notation above the maximal punishment that the players $I \backslash\{i\}$ can inflict to player $i$ is represented by actions in the set $L_{X_{-i}}$, i.e., this is the set containing all the private information strategies of the players $I \backslash\{i\}$. The best player $i$ can do is to maximize her payoff based on her own private information. This leads to the following definitions.

Definition 4.1. The ex-ante minimax point of a strategic game with asymmetric information $G$ is the sequence of extended real numbers $v^{*}=\left(v_{1}^{*}, v_{2}^{*}, \ldots\right)$, where for each player $i$ we have

$$
v_{i}^{*}=\inf _{x_{-i} \in L_{X_{-i}}} \sup _{x_{i} \in L_{X_{i}}} v_{i}\left(x_{-i}, x_{i}\right) .
$$

We shall say that the ex-ante minimax point is attainable, if $v_{i}^{*}$ is attained for each player $i$, i.e.,

$$
v_{i}^{*}=\min _{x_{-i} \in L_{X_{-i}}} \max _{x_{i} \in L_{X_{i}}} v_{i}\left(x_{-i}, x_{i}\right) .
$$

Similarly, we define the interim minimax point for which the actions are made after all players have received their own private information, that is, at an interim stage.

Definition 4.2. The interim minimax point of a strategic game with asymmetric information $G$ is the sequence of extended real valued functions

$V^{*}(\cdot)=\left(V_{1}^{*}(\cdot), V_{2}^{*}(\cdot), \ldots\right)$, where for each player $i$ we have

$$
\begin{aligned}
V_{i}^{*}(\cdot): \Omega & \rightarrow \overline{\mathbb{R}} \\
\omega & \rightarrow V_{i}^{*}(\omega)=\inf _{x_{-i} \in L_{X_{-i}}} \sup _{x_{i} \in L_{X_{i}}} V_{i}\left(\omega, x_{-i}, x_{i}\right) .
\end{aligned}
$$


We shall say that the interim minimax point is attainable, if for each player $i$, we have

$$
\begin{aligned}
V_{i}^{*}(\cdot): \Omega & \rightarrow \overline{\mathbb{R}} \\
\omega & \rightarrow V_{i}^{*}(\omega)=\min _{x_{-i} \in L_{X_{-i}}} \max _{x_{i} \in L_{X_{i}}} V_{i}\left(\omega, x_{-i}, x_{i}\right) .
\end{aligned}
$$

Definitions 4.1 and 4.2 do not take into account the fact that players $I \backslash\{i\}$ may cooperate against player $i$. Even if, an explicit cooperation is not allowed, player $i$ may not know this. Therefore, the worst punishment player $i$ may expect to receive is when the others cooperate against him. This idea can be formalized by assuming that players in $I \backslash\{i\}$, pool their own private information. In other words, the strategy vector $x_{-i}(\cdot)$ is assumed to be $\bigvee_{j \in I \backslash\{i\}} \mathcal{F}_{j}$-measurable.

On the other hand, the least severe punishment that player $i$ may expect to receive is when all the other players use the common knowledge information strategies, that is the strategy vector $x_{-i}(\cdot)$ is $\bigwedge_{j \in I \backslash\{i\}} \mathcal{F}_{j}$-measurable.

To this end, define for each fixed player $i$ and each $j \in I \backslash\{i\}$ the sets ${ }^{4}$

$$
\begin{aligned}
& L_{X_{-i}^{j}}^{p}=\left\{x_{j} \in L_{1}(\mu, Y): \quad x_{j}(\cdot) \text { is } \bigvee_{j \in I \backslash\{i\}} \mathcal{F}_{j}-\text { measurable and } x_{j}(\omega) \in X_{j}(\omega) \mu-\text { a. e. }\right\}, \\
& L_{X_{-i}^{c}}^{c}=\left\{x_{j} \in L_{1}(\mu, Y): \quad x_{j}(\cdot) \text { is } \bigwedge_{j \in I \backslash\{i\}} \mathcal{F}_{j} \text {-measurable and } x_{j}(\omega) \in X_{j}(\omega) \mu-\text { a. e. }\right\} .
\end{aligned}
$$

Let $L_{X_{-i}}^{p}=\prod_{j \in I \backslash\{i\}} L_{X_{-i}^{j}}^{p}$ and $L_{X_{-i}}^{c}=\prod_{j \in I \backslash\{i\}} L_{X_{-i}^{j}}^{c}$.

Observe, that if the players had used the common knowledge information strategies or the private information (i.e., $\mathcal{F}_{j}$ ), then since $L_{X_{-i}}^{c}$ and $L_{X_{-i}}$ are subsets of $L_{X_{-i}}^{p}$, the punishment inflicted to player $i$ would have been less severe. Indeed, the larger the set, the bigger the punishment. Notice that, since for each $i, j \in I$, with $j \neq i$,

$$
L_{X_{-i}}^{c} \subseteq L_{X_{-i}} \subseteq L_{X_{-i}}^{p},
$$

it follows that

$$
\begin{aligned}
\inf _{x_{-i} \in L_{X_{-i}}^{c}} \sup _{x_{i} \in L_{X_{i}}} v_{i}\left(x_{-i}, x_{i}\right) & \geq \inf _{x_{-i} \in L_{X_{-i}}} \sup _{x_{i} \in L_{X_{i}}} v_{i}\left(x_{-i}, x_{i}\right) \\
& \geq \inf _{x_{-i} \in L_{X_{-i}}^{p}} \sup _{i} \in L_{X_{i}} v_{i}\left(x_{-i}, x_{i}\right) .
\end{aligned}
$$

Definitions 4.1 and 4.2 can be formulated in terms of the sets $L_{X_{-i}}^{p}$ and $L_{X_{-i}}^{c}$ and the existence theorem 6.1 and 6.2 remain valid.

\footnotetext{
${ }^{4}$ The apexes "p" and "c" stand respectively for "pool" and "common" information.
} 
As noted above, player $i$ maximizes her payoff using her own private information, i.e., $x_{i}(\cdot)$ is $\mathcal{F}_{i}$-measurable. For each player $i \in I$, we call an action $x$ satisfying (1) an ex-ante optimizer for the ex-ante minimax value $v_{i}^{*}$. Similarly, we call an action $x$ satisfying (2) an interim optimizer for the interim minimax value $V_{i}^{*}$. Clearly optimizers, if they exist, may be different for different players.

The example below shows that if the minimax point is attainable, the sets of ex-ante and interim optimizers may differ.

Example 4.3. Consider the 2-person asymmetric information game with two equiprobable states of nature, that is $\Omega=\left\{\omega_{1}, \omega_{2}\right\}$ and $\mu\left(\omega_{1}\right)=\mu\left(\omega_{2}\right)=\frac{1}{2}$, and where the players' characteristics are given as follows

$$
\begin{aligned}
\mathcal{F}_{1} & =\left\{\left\{\omega_{1}\right\},\left\{\omega_{2}\right\}\right\} \quad \text { and } \mathcal{F}_{2}=\left\{\omega_{1}, \omega_{2}\right\} \\
X_{1}\left(\omega_{1}\right) & =\left[0, \frac{1}{2}\right] ; \quad X_{1}\left(\omega_{2}\right)=[0,1] \quad \text { and } X_{2}\left(\omega_{1}\right)=X_{2}\left(\omega_{2}\right)=[0,1] \\
u_{1}\left(\omega_{1}, x_{1}\left(\omega_{1}\right), x_{2}\left(\omega_{1}\right)\right) & =x_{1}\left(\omega_{1}\right)+x_{2}\left(\omega_{1}\right) \text { and } u_{1}\left(\omega_{2}, x_{1}\left(\omega_{2}\right), x_{2}\left(\omega_{2}\right)\right)=x_{1}\left(\omega_{2}\right)-x_{2}\left(\omega_{2}\right) \\
u_{2}\left(\omega, x_{1}(\omega), x_{2}(\omega)\right) & =x_{1}(\omega)+x_{2}(\omega) \text { for all } \omega \in \Omega .
\end{aligned}
$$

The ex-ante minimax point is attainable; indeed,

$$
\begin{aligned}
v_{1}^{*} & =\min _{x_{2} \in L_{X_{2}}} \max _{x_{1} \in L_{X_{1}}}\left[\frac{1}{2} u_{1}\left(\omega_{1}, x_{1}\left(\omega_{1}\right), x_{2}\left(\omega_{1}\right)\right)+\frac{1}{2} u_{1}\left(\omega_{2}, x_{1}\left(\omega_{2}\right), x_{2}\left(\omega_{2}\right)\right)\right]= \\
& =\min _{x_{2} \in L_{X_{2}}} \max _{x_{1} \in L_{X_{1}}}\left[\frac{1}{2} x_{1}\left(\omega_{1}\right)+\frac{1}{2} x_{2}\left(\omega_{1}\right)+\frac{1}{2} x_{1}\left(\omega_{2}\right)-\frac{1}{2} x_{2}\left(\omega_{2}\right)\right]= \\
& =\max _{x_{1} \in L_{X_{1}}}\left[\frac{1}{2} x_{1}\left(\omega_{1}\right)+\frac{1}{2} x_{1}\left(\omega_{2}\right)\right]=\frac{3}{4}, \quad \text { and similarly } \\
v_{2}^{*} & =1 .
\end{aligned}
$$

Also, the interim minimax point is attainable but it differs form the ex-ante one; indeed,

$$
\begin{aligned}
V_{1}^{*}\left(\omega_{1}\right) & =\min _{x_{2} \in L_{X_{2}}} \max _{x_{1} \in L_{X_{1}}} \frac{\mu\left(\omega_{1}\right)}{\mu\left(\omega_{1}\right)} u_{1}\left(\omega_{1}, x_{1}\left(\omega_{1}\right), x_{2}\left(\omega_{1}\right)\right)= \\
& =\min _{x_{2} \in L_{X_{2}}} \max _{x_{1} \in L_{X_{1}}}\left[x_{1}\left(\omega_{1}\right)+x_{2}\left(\omega_{1}\right)\right]=\frac{1}{2} ; \\
V_{1}^{*}\left(\omega_{2}\right) & =\min _{x_{2} \in L_{X_{2}}} \max _{x_{1} \in L_{X_{1}}} \frac{\mu\left(\omega_{2}\right)}{\mu\left(\omega_{2}\right)} u_{1}\left(\omega_{2}, x_{1}\left(\omega_{2}\right), x_{2}\left(\omega_{2}\right)\right)= \\
& =\min _{x_{2} \in L_{X_{2}}} \max _{x_{1} \in L_{X_{1}}}\left[x_{1}\left(\omega_{2}\right)-x_{2}\left(\omega_{2}\right)\right]=0, \quad \text { and finally }
\end{aligned}
$$




$$
\begin{aligned}
V_{2}^{*}\left(\omega_{1}\right)=V_{2}^{*}\left(\omega_{2}\right) & =\min _{x_{1} \in L_{X_{1}} x_{2} \in L_{X_{2}}} \max _{\mu\left(\omega_{1}, \omega_{2}\right)} u_{2}\left(\omega_{1}, x_{1}\left(\omega_{1}\right), x_{2}\left(\omega_{1}\right)\right)+ \\
& \left.+\frac{\mu\left(\omega_{2}\right)}{\mu\left(\omega_{1}, \omega_{2}\right)} u_{2}\left(\omega_{2}, x_{1}\left(\omega_{2}\right), x_{2}\left(\omega_{2}\right)\right)\right]= \\
& =\min _{x_{1} \in L_{X_{1}}} \max _{x_{2} \in L_{X_{2}}}\left[\frac{1}{2} x_{1}\left(\omega_{1}\right)+\frac{1}{2} x_{2}\left(\omega_{1}\right)+\frac{1}{2} x_{1}\left(\omega_{2}\right)+\frac{1}{2} x_{2}\left(\omega_{2}\right)\right]=1 .
\end{aligned}
$$

Nevertheless, if the payoff functions are not continuous or if the random strategy sets are not compact, the set of ex-ante and interim optimizers may be empty, as the examples in the next section show.

\section{Examples of non-existence of equilibrium}

As in the deterministic case (see [2]), one can easily show that without, either compactness of the strategy sets or continuity of the payoff functions, an equilibrium may not exist.

Example 5.1. Consider the 2-person asymmetric information game with two equiprobable states of nature, that is $\Omega=\left\{\omega_{1}, \omega_{2}\right\}$ and $\mu\left(\omega_{1}\right)=\mu\left(\omega_{2}\right)=\frac{1}{2}$. The players' characteristics are given as follows:

$$
\begin{aligned}
\mathcal{F}_{1} & =\left\{\left\{\omega_{1}\right\},\left\{\omega_{2}\right\}\right\} \quad \text { and } \mathcal{F}_{2}=\left\{\omega_{1}, \omega_{2}\right\} \\
X_{1}\left(\omega_{1}\right) & =(0,+\infty) ; \quad X_{1}\left(\omega_{2}\right)=(0,1) \text { and } X_{2}\left(\omega_{1}\right)=X_{2}\left(\omega_{2}\right)=(0,+\infty) \\
u_{1}\left(\omega_{1}, x_{1}\left(\omega_{1}\right), x_{2}\left(\omega_{1}\right)\right) & =x_{1}\left(\omega_{1}\right)+x_{2}\left(\omega_{1}\right) \text { and } u_{1}\left(\omega_{2}, x_{1}\left(\omega_{2}\right), x_{2}\left(\omega_{2}\right)\right)=x_{1}\left(\omega_{2}\right)-x_{2}\left(\omega_{2}\right) \\
u_{2}\left(\omega, x_{1}(\omega), x_{2}(\omega)\right) & =x_{1}(\omega)+x_{2}(\omega) \text { for all } \omega \in \Omega .
\end{aligned}
$$

It is easy to see that the ex-ante minimax point is as follows

$$
\begin{aligned}
v_{1}^{*} & =\inf _{x_{2} \in L_{X_{2}}} \sup _{x_{1} \in L_{X_{1}}}\left[\frac{1}{2} u_{1}\left(\omega_{1}, x_{1}\left(\omega_{1}\right), x_{2}\left(\omega_{1}\right)\right)+\frac{1}{2} u_{1}\left(\omega_{2}, x_{1}\left(\omega_{2}\right), x_{2}\left(\omega_{2}\right)\right)\right]= \\
& =\inf _{x_{2} \in L_{X_{2}}} \sup _{x_{1} \in L_{X_{1}}}\left[\frac{1}{2} x_{1}\left(\omega_{1}\right)+\frac{1}{2} x_{2}\left(\omega_{1}\right)+\frac{1}{2} x_{1}\left(\omega_{2}\right)-\frac{1}{2} x_{2}\left(\omega_{2}\right)\right]= \\
& =\sup _{x_{1} \in L_{X_{1}}}\left[\frac{1}{2} x_{1}\left(\omega_{1}\right)+\frac{1}{2} x_{1}\left(\omega_{2}\right)\right]=+\infty, \quad \text { and similarly } \\
v_{2}^{*} & =+\infty
\end{aligned}
$$


It is easy to see that the interim minimax point is as follows

$$
\begin{aligned}
V_{1}^{*}\left(\omega_{1}\right) & =\inf _{x_{2} \in L_{X_{2}}} \sup _{x_{1} \in L_{X_{1}}} \frac{\mu\left(\omega_{1}\right)}{\mu\left(\omega_{1}\right)} u_{1}\left(\omega_{1}, x_{1}\left(\omega_{1}\right), x_{2}\left(\omega_{1}\right)\right)= \\
& =\inf _{x_{2} \in L_{X_{2}}} \sup _{x_{1} \in L_{X_{1}}}\left[x_{1}\left(\omega_{1}\right)+x_{2}\left(\omega_{1}\right)\right]=+\infty ; \\
V_{1}^{*}\left(\omega_{2}\right) & =\inf _{x_{2} \in L_{X_{2}}} \sup _{x_{1} \in L_{X_{1}}} \frac{\mu\left(\omega_{2}\right)}{\mu\left(\omega_{2}\right)} u_{1}\left(\omega_{2}, x_{1}\left(\omega_{2}\right), x_{2}\left(\omega_{2}\right)\right)= \\
& =\inf _{x_{2} \in L_{X_{2}}} \sup _{x_{1} \in L_{X_{1}}}\left[x_{1}\left(\omega_{2}\right)-x_{2}\left(\omega_{2}\right)\right]=-\infty, \quad \text { and finally } \\
V_{2}^{*}\left(\omega_{1}\right)=V_{2}^{*}\left(\omega_{2}\right) & =\inf _{x_{1} \in L_{X_{1}} x_{2} \in L_{X_{2}}} \sup _{\mu\left(\omega_{1}, \omega_{2}\right)}\left[\frac{\mu\left(\omega_{1}\right)}{\mu\left(\omega_{1}, x_{1}\left(\omega_{1}\right), x_{2}\left(\omega_{1}\right)\right)+}\right. \\
& \left.+\frac{\mu\left(\omega_{2}\right)}{\mu\left(\omega_{1}, \omega_{2}\right)} u_{2}\left(\omega_{2}, x_{1}\left(\omega_{2}\right), x_{2}\left(\omega_{2}\right)\right)\right]= \\
& =\inf _{x_{1} \in L_{X_{1}} x_{2} \in L_{X_{2}}} \sup _{2}\left[\frac{1}{2} x_{1}\left(\omega_{1}\right)+\frac{1}{2} x_{2}\left(\omega_{1}\right)+\frac{1}{2} x_{1}\left(\omega_{2}\right)+\frac{1}{2} x_{2}\left(\omega_{2}\right)\right]=+\infty
\end{aligned}
$$

Therefore, since the random strategy sets are not compact, even if the random payoff functions are continuous, the set of ex-ante as well as interim optimizers is empty.

The example below shows that the continuity assumption of the payoff functions cannot be dispensed with.

Example 5.2. Consider the 2-person asymmetric information game with two equiprobable states of nature, that is $\Omega=\left\{\omega_{1}, \omega_{2}\right\}$ and $\mu\left(\omega_{1}\right)=\mu\left(\omega_{2}\right)=\frac{1}{2}$, and where the players' characteristics are given as follows

$$
\begin{aligned}
\mathcal{F}_{1} & =\left\{\left\{\omega_{1}\right\},\left\{\omega_{2}\right\}\right\} \quad \text { and } \mathcal{F}_{2}=\left\{\omega_{1}, \omega_{2}\right\} \\
X_{1}\left(\omega_{1}\right) & =\left[0, \frac{1}{2}\right] ; \quad X_{1}\left(\omega_{2}\right)=\left[\frac{1}{2}, 1\right] \quad \text { and } X_{2}\left(\omega_{1}\right)=X_{2}\left(\omega_{2}\right)=[0,1] .
\end{aligned}
$$

Thus, the random strategy sets are compact, but the payoff functions are not continuous and defined by

$$
\begin{gathered}
u_{1}\left(\omega_{1}, x_{1}\left(\omega_{1}\right), x_{2}\left(\omega_{1}\right)\right)= \begin{cases}x_{1}\left(\omega_{1}\right) & \text { if } x_{1}\left(\omega_{1}\right) \in\left[0, \frac{1}{4}\right) \\
-x_{1}\left(\omega_{1}\right) & \text { if } x_{1}\left(\omega_{1}\right) \in\left[\frac{1}{4}, \frac{1}{2}\right],\end{cases} \\
u_{1}\left(\omega_{2}, x_{1}\left(\omega_{2}\right), x_{2}\left(\omega_{2}\right)\right)= \begin{cases}x_{1}\left(\omega_{2}\right) & \text { if } x_{1}\left(\omega_{2}\right) \in\left[\frac{1}{2}, \frac{3}{4}\right) \\
-x_{1}\left(\omega_{2}\right) & \text { if } x_{1}\left(\omega_{2}\right) \in\left[\frac{3}{4}, 1\right], \text { finally for all } \omega \in \Omega\end{cases} \\
u_{2}\left(\omega, x_{1}(\omega), x_{2}(\omega)\right)= \begin{cases}x_{2}(\omega) & \text { if } x_{2}(\omega) \in(0,1) \\
\frac{1}{2} & \text { if } x_{2}(\omega) \in\{0,1\} .\end{cases}
\end{gathered}
$$


It is easy to see that the ex-ante minimax point is as follows

$$
v_{1}^{*}=\frac{1}{2} \quad \text { and } \quad v_{2}^{*}=1
$$

while for the interim minimax point, we have

$$
\begin{aligned}
V_{1}^{*}\left(\omega_{1}\right) & =\frac{1}{4} \\
V_{1}^{*}\left(\omega_{2}\right) & =\frac{3}{4} \text { and } \\
V_{2}^{*}\left(\omega_{1}\right)=V_{2}^{*}\left(\omega_{2}\right) & =1
\end{aligned}
$$

Hence, the set of ex-ante as well as interim optimizers is empty.

By imposing compactness and continuity in the weak topology, we will be able to prove the existence of an ex-ante and an interim minimax point in the next section.

\section{Existence Theorems}

\subsection{Assumptions}

We now list the main assumptions needed to prove that an ex-ante as well as an interim minimax point is attainable.

(A.1) For each $i, X_{i}: \Omega \rightarrow Y$ is $\mathcal{F}_{i}$-lower measurable, non-empty, integrably bounded, closed, weakly compact and convex valued correspondence,

(A.2) For each $x \in \prod_{i \in I} Y$ and for each player $i \in I, u_{i}(\cdot, x): \Omega \rightarrow \mathbb{R}$ is $\mathcal{F}$-measurable. Moreover, for all $\omega$ and for all $i, u_{i}(\omega, \cdot): \prod_{i \in I} Y \rightarrow \mathbb{R}$ is weakly jointly continuous and integrably bounded.

\subsection{Theorems}

Theorem 6.1. Assume that (A.1) and (A.2) hold. Then the ex-ante minimax point is attainable.

Theorem 6.2. Assume that (A.1) and (A.2) hold. Then the interim minimax point is attainable.

Clearly, the theorem in the deterministic case, proved by Aliprantis et al. in [2], can be viewed as a corollary of ours. Indeed, in the special case of full information, i.e., when the private information of each player is represented by the $\sigma$-algebra of all singletons, the 
interim expected payoff function reduces to be the ex-post one. Therefore, theorem 6.2 includes as a special case a version of the existence of a deterministic minimax point, in $[2]$.

\subsection{Proof of the Theorem 6.1}

First, we observe that:

Claim 6.3. If (A.1) holds, then $L_{X}$ is non-empty and weakly compact.

Proof: We first prove that $L_{X}$ is non-empty. Since, for each fixed $i, X_{i}$ is $\mathcal{F}_{i}$-measurable, all the conditions of Kuratowski and Ryll-Nardzewski Measurable Selection Theorem (see [1] p. 600) are satisfied and hence there exists an $\mathcal{F}_{i}$-measurable function $x_{i}^{*}: \Omega \rightarrow Y$ such that $x_{i}^{*}(\omega) \in X_{i}(\omega)$ for almost all $\omega \in \Omega$. Therefore, we just need to show that $x_{i}^{*} \in L_{1}(\mu, Y)$, that is $x_{i}^{*}$ is a Bochner integrable function. But this follows directly from the assumption that $X_{i}$ is integrably bounded. Thus, for all $i, L_{X_{i}}$ is non-empty, and so is $L_{X}=\prod_{i \in I} L_{X_{i}}$.

We are now ready to prove that $L_{X}$ is weakly compact. First, notice that for all $i \in I$, $L_{X_{i}}$ is a weakly closed subset of the weakly compact set $\left\{x_{i} \in L_{1}(\mu, Y): x_{i}(\omega) \in\right.$ $X_{i}(\omega)$ for almost all $\left.\omega \in \Omega\right\}$, (recall Diestel's theorem, see [8]). Therefore, for each fixed $i$, $L_{X_{i}}$ is weakly compact, since it is weakly closed subset of a weakly compact set. Consequently, the set $L_{X}=\prod_{i \in I} L_{X_{i}}$ is also weakly compact by Tychonoff's Theorem.

Claim 6.4. Assume that (A.1) and (A.2) hold, then for each $i$ and $\omega$, the functions $v_{i}(\cdot)$ and $V_{i}(\omega, \cdot)$ are weakly continuous.

Proof: See Yannelis (1991, p.191).

We can now complete the proof of the theorem by applying the Berge Maximum Theorem, which obviously holds for a continuous correspondence between two general topological spaces, in particular it holds with the weak topology. For each fixed player $i$, consider the constant correspondence $\Phi_{i}: L_{X_{-i}} \rightarrow 2^{L_{X_{i}}}$ defined by

$$
\phi_{i}\left(x_{-i}\right)=L_{X_{i}} \quad \forall x_{-i} \in L_{X_{-i}} .
$$

Obviously $\Phi_{i}(\cdot)$ is weakly continuous (because it is constant), non-empty and weakly compact-valued correspondence (by Claim 6.3).

Notice that the graph of $\Phi_{i}$ coincides with $L_{X}$. In fact:

$$
\begin{aligned}
& G r_{\Phi_{i}}=\left\{\left(x_{-i}, x_{i}\right) \in L_{X_{-i}} \times L_{X_{i}}: \quad x_{i} \in \Phi_{i}\left(x_{-i}\right)\right\}= \\
& =\left\{\left(x_{-i}, x_{i}\right) \in L_{X_{-i}} \times L_{X_{i}}: \quad x_{i} \in L_{X_{i}}\right\}= \\
& =L_{X_{-i}} \times L_{X_{i}}=L_{X} \text {. }
\end{aligned}
$$


Define the function $f_{i}: G r_{\Phi_{i}}=L_{X} \rightarrow \mathbb{R}$ by

$$
f_{i}\left(x_{-i}, x_{i}\right)=v_{i}\left(x_{-i}, x_{i}\right) .
$$

By Claim $6.4, f_{i}(\cdot)$ is weakly continuous.

From Claims 6.3 and 6.4 it follows that all conditions of the Berge Maximum Theorem are satisfied and hence the value function $m_{i}: L_{X_{-i}} \rightarrow \mathbb{R}$ defined by

$$
\begin{aligned}
m_{i}\left(x_{-i}\right) & =\max _{x_{i} \in \Phi_{i}\left(x_{-i}\right)} f_{i}\left(x_{-i}, x_{i}\right)= \\
& =\max _{x_{i} \in L_{X_{i}}} v_{i}\left(x_{-i}, x_{i}\right)
\end{aligned}
$$

is weakly continuous and the correspondence $\mu_{i}: L_{X_{-i}} \rightarrow 2^{L_{X_{i}}}$ of maximizers, defined by

$$
\mu_{i}\left(x_{-i}\right)=\left\{x_{i} \in L_{X_{i}}: v_{i}\left(x_{-i}, x_{i}\right)=m_{i}\left(x_{-i}\right)\right\}
$$

has non-empty and weakly compact values.

Thus, by virtue of the weak compactness of set the $L_{X_{-i}}$, the weakly continuous function $m_{i}$ attains its minimum over $L_{X_{-i}}$.

\subsection{Proof of the Theorem 6.2}

The proof is in the same spirit of the Theorem 6.1. It suffices to define the functions used in the proof of the previous theorem as follows: for all $\omega \in \Omega$, define $g_{i}(\omega, \cdot): L_{X_{-i}} \times L_{X_{i}} \rightarrow \mathbb{R}$ by

$$
g_{i}\left(\omega, x_{-i}, x_{i}\right)=V_{i}\left(\omega, x_{-i}, x_{i}\right) .
$$

The value function $m_{i}(\omega, \cdot): L_{X_{-i}} \rightarrow \mathbb{R}$ is defined, for all $\omega \in \Omega$, by

$$
\begin{aligned}
m_{i}\left(\omega, x_{-i}\right) & =\max _{x_{i} \in \Phi_{i}\left(x_{-i}\right)} g_{i}\left(\omega, x_{-i}, x_{i}\right)= \\
& =\max _{x_{i} \in L_{X_{i}}} V_{i}\left(\omega, x_{-i}, x_{i}\right) .
\end{aligned}
$$

The correspondence $\mu_{i}(\omega, \cdot): L_{X_{-i}} \rightarrow 2^{L_{X_{i}}}$ of maximizers is defined, for all $\omega \in \Omega$, by

$$
\mu_{i}\left(\omega, x_{-i}\right)=\left\{x_{i} \in L_{X_{i}}: \quad V_{i}\left(\omega, x_{-i}, x_{i}\right)=m_{i}\left(\omega, x_{-i}\right)\right\} .
$$

One can follow the steps of the previous theorem to conclude the proof.

Open question: The separability assumption plays an important role to apply the $\mathrm{Ku}$ ratowski and Ryll-Nardzewski Measurable Selection Theorem. We do not know if the existence theorem can be proved without the separability assumption on the strategy space. 


\section{References}

[1] Aliprantis, C.D., Border, K.C., 2006. Infinite Dimensional Analysis: A Hitchhikers Guide, third edition. SpringerVerlag, New York.

[2] Aliprantis, C.D., Chakrabarti S. K., Topolyan, I., 2009. A proof of the existence of the minimax point of a strategic game, Economics Letters, doi:10.1016/j.econlet.2009.08.015

[3] Castaing, C., Valadier, M., 1977. Convex analysis and measurable multifunctions. In: Lect. Notes Math., vol. 588. Berlin: Springer.

[4] Diestel J, Uhl JJ, 1977. Vector measures. Mathematical surveys, no. 15. American Mathematical Society, Providence Rhode Island.

[5] Fudenberg, D., Maskin, E., 1986. The folk theorem in repeated games with discounting or with incomplete information. Econometrica 54, 533-554.

[6] Myerson, R.B., 1991. Game Theory: Analysis of Conflict. Harvard University Press. Cambridge, MA.

[7] Thomas, J.P., 1995. Subgame-perfect attainment of minimax punishments in discounted two-person games, Economics Letters 47, 1-4

[8] Yannelis, N.C., 1991. The core of an economy with differential information. Economic Theory 1, 183-198.

[9] Yannelis, N.C. and Rustichini, A., 1991. On the existence of Correlated Equilibria, in Equilibrium Theory in Infinite Dimensional Space, M. Ali Khan and Nicholas C. Yannelis (eds.), Springer- Verlag. 